\title{
A Double-Edged Sword and a Golden Opportunity for Healthcare Epidemiology
}

\author{
Robert A. Weinstein, MD; David K. Henderson, MD
}

The field of hospital epidemiology is at a fascinating and challenging crossroad. The past decade has witnessed an explosion of interest in healthcare quality, adverse events and medical errors in health care, as well as an intense interest in the spiraling costs associated with the delivery of healthcare in the United States. The Institute of Medicine of the National Academy of Sciences published an initial report in 1999 suggesting that thousands of US citizens die unnecessarily each year from medical errors. ${ }^{1}$ A second Institute of Medicine report, published in 2004, identified an effective road map for addressing some of the complex issues contributing to what the Institute of Medicine referred to as a "quality chasm."

Healthcare-associated infections have been identified as significant contributors to unnecessary morbidity and mortality associated with the delivery of health care in the United States and have begun to receive increasing scrutiny over the past 5 or 6 years. More recently, the Government Accountability Office issued a report noting that healthcare-associated infections, taken together, are estimated to be one of the top 10 causes of death in the United States. ${ }^{3}$ In a separate report, the Government Accountability Office recommended that the Department of Health and Human Services display additional leadership in prioritizing recommended prevention practices and in improving the collection and use of nationwide data about healthcare-associated infections. ${ }^{4}$ In response, the Secretary of the Department of Health and Human Services, his principal Deputy, and the Acting Assistant Secretary for Health convened a department-wide task force to develop a national action plan for healthcare-associated infections.

Individual states also have taken an interest in the problem: 25 states now require public reporting of healthcare-associated infections; the majority of the remaining states are considering such legislation. Most recently, the Center for Medicare and Medicaid Services announced that, beginning October 1, 2008, Medicare will no longer pay the costs associated with infections resulting from a hospital stay (ie, they will not provide coverage for infections that were not present at admission). ${ }^{5,6}$

Thus, for the first time in both our lengthy careers in the field, the discipline of healthcare epidemiology has become "high profile." Such intense interest is a double-edged sword, with stakeholders providing a great deal of input regarding what to do, as well as when and how to improve our professional practices.

The Society for Healthcare Epidemiology of America (SHEA) has been an active participant in nearly all of the high-level discussions relating to healthcare-associated infections and has recently published (in collaboration with the Infectious Diseases Society of America [IDSA]) a compendium of strategies on how to prevent healthcare-associated infections in acute care hospitals. ' The SHEA-IDSA compendium of strategies complement the guidelines that have been issued by the Department of Health and Human Services Secretary's Healthcare Infection Control Practices Advisory Committee. When examined carefully, these strategies identify what we know and what we don't know about the epidemiology, pathogenesis, transmission, and prevention of transmission of healthcare-associated infections.

Whereas such focus on the most important issues in the field of healthcare epidemiology delivers a heretofore unprecedented level of scrutiny of the contributions of hospital epidemiology programs to patient safety, it also provides a unique opportunity for the field to advance. So, what are our goals and how do we attain them? The last several years have seen an increased emphasis on health services research - studies that have often demonstrated how the systematic implementation of approaches that we already know or suspect can effectively reduce risks for transmission of healthcareassociated infections can beneficially influence patient outcomes. The statewide study of Pronovost and his colleagues, ${ }^{8,9}$ for example, demonstrated conclusively that rigorously implementing a bundle of previously identified successful strategies can result in substantially improved outcomes. Whereas

From the Division of Medicine, Stroger Hospital of Cook County, and Rush Medical College, Chicago, Illinois (R.A.W.), and the National Institutes of Health, Bethesda, Maryland (D.K.H.). Dr. Weinstein and Dr. Henderson are members of the Research Committee of the Society for Healthcare Epidemiology of America (SHEA), and authored this editorial on behalf of that committee and as an official statement of SHEA.

Received November 24, 2008; accepted November 24, 2008; electronically published December 9, 2008.

Infect Control Hosp Epidemiol 2009;30:1-3

(C) 2008 by The Society for Healthcare Epidemiology of America. All rights reserved. 0899-823X/2009/3001-3001\$15.00. DOI: 10.1086/596559 
these approaches have been incredibly useful to healthcare institutions across the country, they can only optimize what we previously have learned from basic and epidemiological studies. An important constituent of these studies is the rigorous measurement of performance and outcomes. These measurement systems must be carefully designed and must include the potential for detecting salutary, as well as unanticipated, untoward consequences. ${ }^{10}$ Successful implementation of this approach-basing healthcare demonstration projects on interventions already tested by high-quality controlled trials-will allow physicians, nurses, and other healthcare professionals to practice "the medicine of 2008" optimally and to improve patient care outcomes substantially.

To achieve further successes, however, we will need a substantial investment in basic science, translational medicine, and epidemiology. Compared with some other disciplines in infectious diseases and microbiology, healthcare epidemiology has far less-solid scientific underpinnings. We simply must conduct the studies that characterize the epidemiology of endemic healthcare-associated infections at a granular level, to provide a clear understanding of which risk factors operate in which settings and which interventions are more, or even most, likely to be successful. In an era when we are beginning to discuss the benefits of "personalized medicine," a broad approach will not lend substantial insight into the myriad complex issues that actually define the epidemiology and pathogenesis of healthcare-associated infections. A broadbrush approach will not allow us to generate new hypotheses that will result in the development of successful interventional strategies for healthcare-associated infections. For example, despite the fact that molecular studies have characterized Clostridium difficile in excruciating detail, even in the year 2008 we have a remarkably limited understanding of the factors that influence the spread of $C$. difficile in the healthcare environment. Do the mechanisms of transmission vary by patient population or institutional demographics? What is the role of immunosuppression? What is the role of antimicrobial stewardship in the institution? Can specific antimicrobials or antimicrobial classes be definitively incriminated? What is the contribution of the hospital inanimate environment to the spread of these organisms? What are the benefits or drawbacks of alternate cleaning strategies? How important is hand hygiene to the prevention of transmission in differing institutional settings? Whereas these are complex, multifactorial questions, they must be answered for us to achieve the kinds of prevention success that we all desire. Hundreds of basic, translational, and epidemiological questions need to be answered for us to be able to intervene, both effectively and with precision, to prevent healthcare-associated infections.

How is it possible that we could find ourselves in this situation in the 21st century, and what can we do to address these issues? First, we have to be able to identify what we know, what we don't know, and, most importantly, what we need to know to be able to intervene successfully. Simply put, we have to identify the questions that need to be addressed; we must design and implement studies that can provide the scientific foundation for the generation of new prevention hypotheses; and we must test these prevention strategies by carefully designed, basic, translational, epidemiological and behavioral studies that are powered adequately to address these questions and to make distinctions definitively.

SHEA recently has created a Research Committee whose mission is to help define the scientific agenda for the Society and for the field of healthcare epidemiology and to develop strategies for addressing this agenda. The Society is currently surveying our membership to assess priorities for our science agenda, as well as the willingness of members and members' institutions to provide resources to support a research consortium. Data from the survey are too preliminary for conclusions, but we are encouraged that many Society members and their respective institutions appear to be willing to assist in supporting a collaborative approach.

Whereas the literature is replete with a variety of studies using quasi-experimental design and methodology, such a consortium-style approach may make it possible to address individual questions in a definitive manner. In addition, the SHEA membership possesses substantial expertise in the design and conduct of complex epidemiological studies, and this expertise can be brought to bear on study design, implementation, and data analysis.

In order for the Society to be able to address these complex questions, we also need a robust measurement infrastructure and a capacity for broad-based data accrual and data analysis. The Centers for Disease Control and Prevention-for decades a national leader in designing, conducting, and funding such studies-has established the National Healthcare Safety Network to provide a repository for epidemiological data. ${ }^{11,12}$ The National Healthcare Safety Network offers a nearly ideal data collection and management infrastructure for the support of the kinds of epidemiological studies that must be conducted to answer important outstanding scientific questions in healthcare epidemiology. And, fortunately, it is already operating and is fully functional.

The draft Department of Health and Human Services national action plan identified several "science gaps" that must be filled for our discipline to design and implement the next set of interventions that can then be bundled and tested. We believe that the development of a science-based, collaborative approach to these questions offers the best chance for success. Further, we believe that complementary activities, beginning with basic, translational, and epidemiological studies, ultimately combined with the efforts of behavioral and health services scientists, offer the best chance for moving us closer to our ultimate goal of "zero" healthcare-associated infections.

The current scrutiny of healthcare-associated infections in our society offers a golden opportunity for us to work together to set priorities, to identify the important scientific questions that must be addressed, to design and implement the studies that can address those questions, and then to work 
collaboratively to achieve this goal. SHEA and its membership should lead these activities for the United States.

Address reprint requests to David K. Henderson, MD, National Institutes of Health, Warren G. Magnuson Clinical Center, Bldg. 10, Rm. 6-1480, 10 Center Drive MSC 1504, Bethesda, MD 20892-1504 (dkh@nih.gov).

\section{REFERENCES}

1. Kohn LT, Corrigan JM, Donaldson MS, eds. To Err Is Human: Building a Safer Health System. National Academy Press; 1999. Washington, DC: Institute of Medicine of the National Academy of Sciences; 1999.

2. Committee on Quality of Health Care in America. Crossing the Quality Chasm: A New Health System for the 21st Century. Washington, DC: Institute of Medicine of the National Academy of Sciences; 2001.

3. Bascetta C. Health-Care-Associated Infections in Hospitals: Number Associated With Medical Devices Unknown, but Experts Report Provider Practices as a Significant Factor. Washington, DC: US Government Accountability Office; 2008.

4. Bascetta CA. Healthcare-Associated Infections in Hospitals: Leadership Needed from HHS to Prioritize Prevention Practices and Improve Data on These Infections. Washington, DC: US Government Accountability Office; 2008.

5. Center for Medicare and Medicaid Services. Notice of Proposed RuleMaking-42 CFR Parts 411, 412, 413, and 489 Medicare Program; Proposed Changes to the Hospital Inpatient Prospective Payment Systems and Fiscal Year 2008 Rates; Proposed Rule. Federal Register 2007; 72(85): 24680-25134.

6. Centers for Medicare and Medicaid Services. Medicare program: changes to the hospital inpatient prospective payment systems and fiscal year 2009 rates; payments for graduate medical education in certain emergency situations; changes to disclosure of physician ownership in hospitals and physical self-referral rules; updates to the long-term care prospective payment system; updates to certain IPPS-excluded hospitals; and collection of information regarding financial relationships between hospitals; final rule. Federal Register 2008; 73(161):48434-49083.

7. Yokoe DS, Mermel LA, Anderson DJ, et al. A compendium of strategies to prevent healthcare-associated infections in acute care hospitals. Infect Control Hosp Epidemiol 2008; 29:S12-S21.

8. Pronovost $\mathrm{P}$, Needham $\mathrm{D}$, Berenholtz $\mathrm{S}$, et al. An intervention to decrease catheter-related bloodstream infections in the ICU. $N$ Engl J Med 2006; 355:2725-2732.

9. Pronovost PJ, Berenholtz SM, Goeschel C, et al. Improving patient safety in intensive care units in Michigan. J Crit Care 2008; 23:207-221.

10. Graves N, McGowan JE, Jr. Nosocomial infection, the Deficit Reduction Act, and incentives for hospitals. JAMA 2008; 300:1577-1579.

11. Edwards JR, Peterson KD, Andrus ML, Dudeck MA, Pollock DA, Horan TC. National Healthcare Safety Network (NHSN) Report, data summary for 2006 through 2007, issued November 2008. Am I Infect Control 2008; 36:609-626.

12. Hidron AI, Edwards JR, Patel J, et al. NHSN annual update: antimicrobial-resistant pathogens associated with healthcare-associated infections: annual summary of data reported to the National Healthcare Safety Network at the Centers for Disease Control and Prevention, 2006-2007. Infect Control Hosp Epidemiol 2008; 29:996-1011. 